\title{
RESENA HISTORICA
}

\section{SOBRE LA NECESIDAD EPISTEMOLÓGICA DE LA INVESTIGACIÓN CUALITATIVA EN SALUD.}

\section{THE EPISTEMOLOGICAL NEED FOR QUALITATIVE} RESEARCH

Manuel Segura -Balbuena (1); Ángela Cejudo-López ${ }^{(2)}$; Eugenia Gil-García ${ }^{(3)}$; Jose Manuel Santos ${ }^{(4)}$; Manuel Ortega-Calvo ${ }^{(5)}$

(1) Enfermero de Familia. Centro de Salud "Esperanza Macarena" (Sevilla)

(2) Enfermera Gestora de Casos. Centro de Salud "Los Bermejales" (Sevilla).

(3) Profesora del Departamento de Enfermería. Universidad de Sevilla.

(4) Médico de Familia. Profesor Asociado del Departamento de Medicina Universidad de Sevilla. Centro de Salud "San Pablo". Unidad de Investigación del Distrito Sanitario At. Primaria (Sevilla). CIBER - OBN CB06 / 03.

(5) Médico de Familia. Centro Salud "Esperanza Macarena". Unidad de Investigación del Distrito Sanitario de At. Primaria (Sevilla). CIBER - OBN CB06 / 03.

Correspondencia: 106mayorque104@gmail.com 


\section{TÍTULO:}

Sobre la necesidad epistemológica de la investigación cualitativa en salud.

\section{RESUMEN}

La sociedad occidental ha aceptado el concepto matemático de número sin ninguna crítica prácticamente desde la Escuela Pitagórica. Los trabajos de Georg Cantor sobre el concepto de infinito y sobre la teoría de conjuntos y las investigaciones lógicas de Kurt Gödel sobre la incompletitud han zarandeado la claridad de la aritmética elemental. Estas investigaciones junto con las discusiones metodológicas entre frecuencialistas y bayesianos en la estadística epidemiológica y los requerimientos aplicativos de la salud basada en la evidencia hacen necesaria la aportación de la investigación cualitativa para el análisis de la realidad sanitaria. Es deseable asimismo la definición epistemológica dentro de cada proyecto cualitativo.

\section{Palabras Clave:}

epistemología, investigación cualitativa, investigación cuantitativa, historia de la ciencia.

\section{Title:}

The epistemological need for qualitative research.

\section{ABSTRACT.}

Western society has accepted the mathematical concept of number uncritically almost from the Pythagorean School. Georg Cantor's work on the concept of infinity and on set theory and Kurt Gödel's logic investigations on incompleteness has shaken clarity of numeracy. These inquiries together with methodological discussions between frecuentists and Bayesian statisticians and applications requirements of evidence-based medicine make necessary the contribution of qualitative research for the analysis of reality of health. It is also desirable epistemological definition within each qualitative project.

\section{Key-words:}

knowledge, qualitative research, research, 20th century history. 


\section{INTRODUCCIÓN}

Al final de su vida, el matemático indio Srinavasa Ramanujan ${ }^{(1)}$ (18871920) estaba ingresado en un hospital londinense ${ }^{(2)}$. En pleno invierno, su amigo y también matemático, G.H. Hardy le hizo una visita. Atenazado por el momento y con timidez, no tuvo otra ocurrencia que comentar el número del taxi que lo había llevado hasta allí, el 1.729. "No creo que sea un número muy interesante, quizás sea una premonición" susurró levemente. "Todo lo contrario" respondió Ramanujan "es el número más pequeño que se puede expresar como la suma de dos cubos de dos formas diferentes" (Figura no 1 )

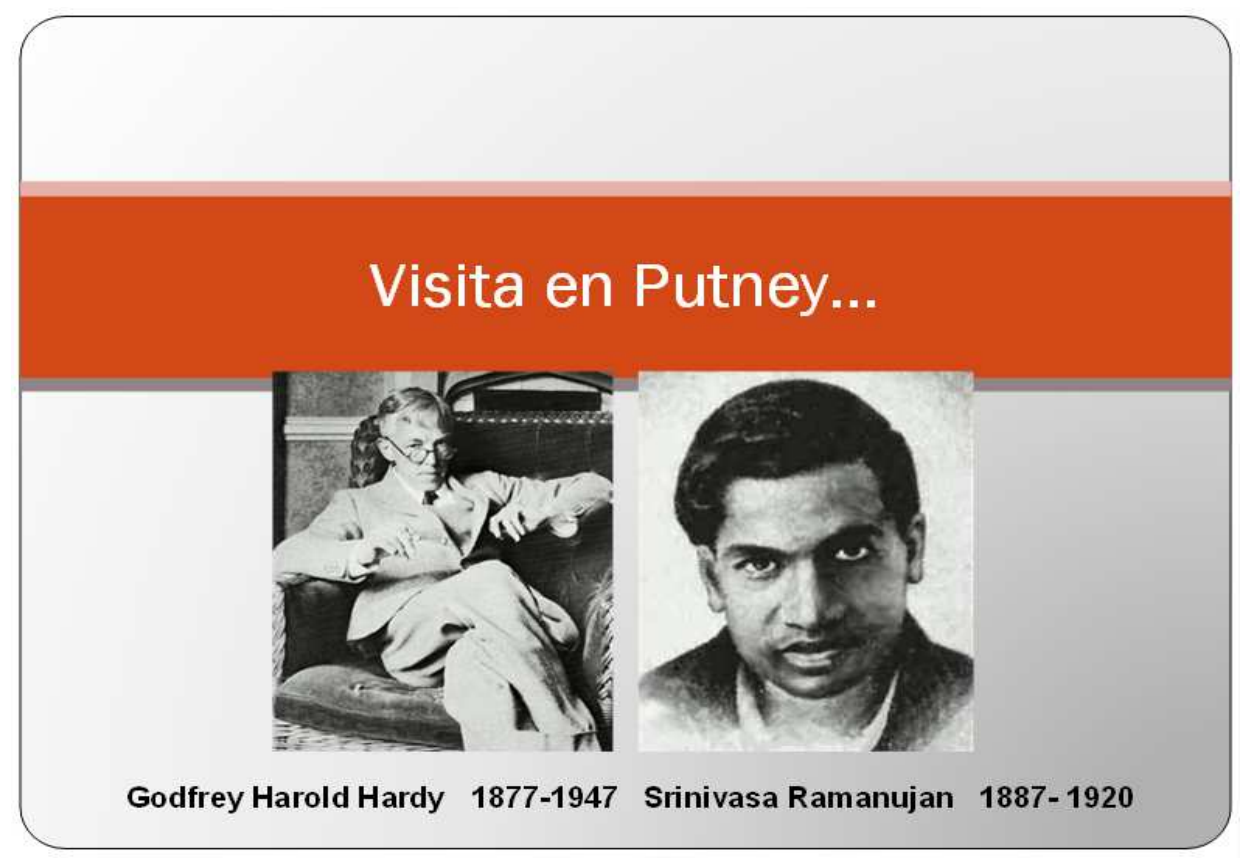

Figura $n^{0} 1$.

Godfrey Hardy y Srinivasa Ramanujan, dos matemáticos que coincidieron a principios del siglo XX en la Inglaterra Postvictoriana.

Efectivamente: $1729=1^{3}+12^{3}=9^{3}+10^{3}$. Ningún número menor tiene esa propiedad. La anécdota es muy famosa y cualquier matemático disfruta contándola no sabiendo realmente muy bien porqué. En el mundo contemporáneo parece que disfrutamos de una especie de idolatría hacia el número. 


\section{La Escuela Pitagórica.}

Al igual que Ramanujan, los pitagóricos se sentían cautivados por la inagotable variedad de números naturales y en especial por sus peculiaridades matemáticas. Estaban fascinados por el 1, 3, 6 y el 10, porque estos números podían expresarse geométricamente en triángulos compuestos de puntos. Entendían a la perfección la importancia de los números que solamente son divididos por sí mismos y por 1 - los números primos como el 2, 3, 5, 7 y el 11. Los números primos se distribuían como piedras preciosas entre la panoplia de números ordinarios. Descubrieron también que ciertos números, como el 6, 28 y el 496 pueden expresarse como la suma de sus divisores. Vestidos de blanco, en cuclillas y agotando el sebo que goteaba de los candiles, buscaban relaciones amistosas entre los números.

En alguna ocasión los pitagóricos escribieron "El número es la esencia de todas las cosas" (Figura no 2)

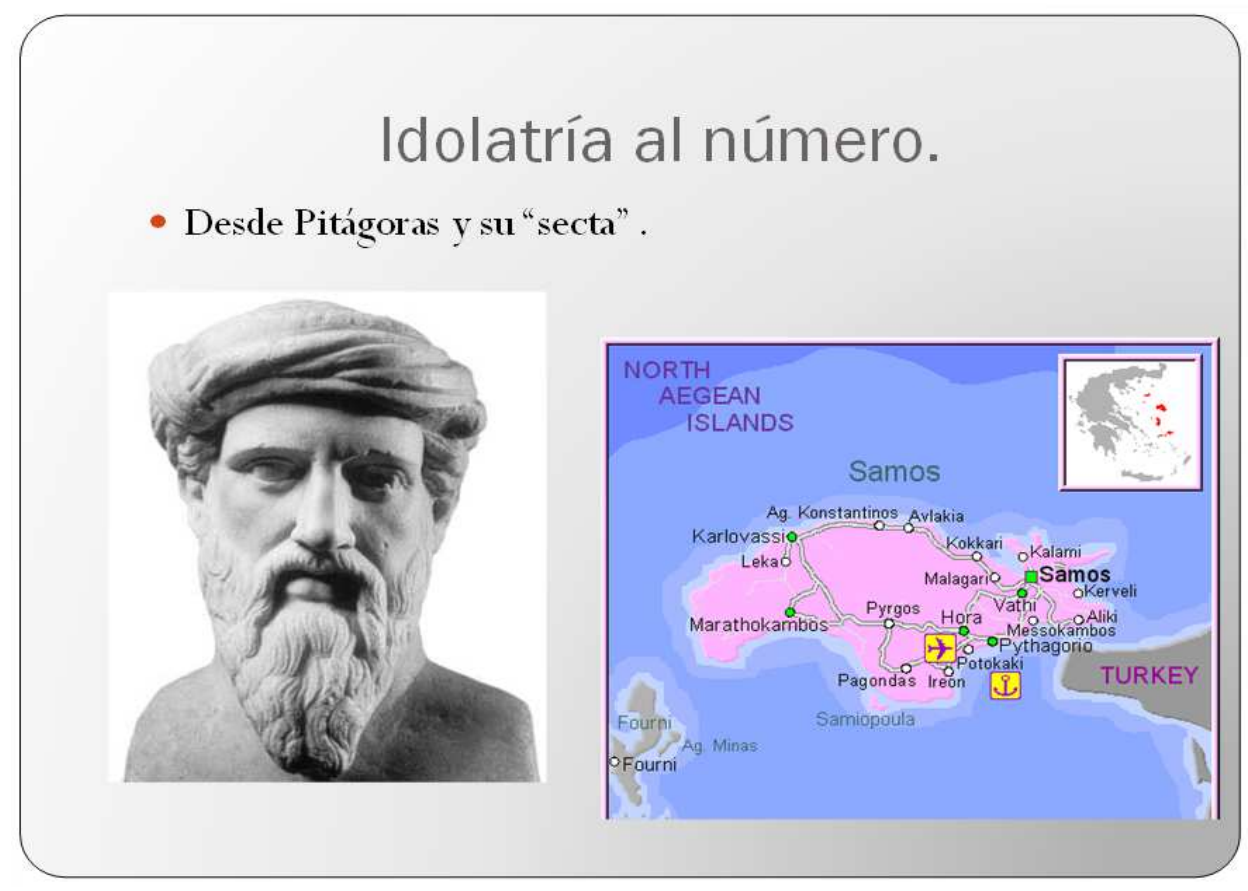

Figura no 2.

Pitágoras nació en la isla de Samos y es considerado el primer matemático puro. 


\section{Epistemología del Número.}

El concepto de número había nacido en la prehistoria cuando el hombre primitivo cuantificaba con las partes de su propio cuerpo. En la escritura cuneiforme existen representaciones de objetos y de su contabilidad. El sistema jónico en la Grecia clásica combinaba las letras del alfabeto griego con algunos otros signos para tareas de cuantificación. La numeración romana es más conocida en occidente y forma parte de nuestra educación primaria. La numeración que se emplea en la actualidad es de origen indo-arábigo. Leonardo Fibonacci también conocido como Leonardo de Pisa(3) introdujo la numeración arábiga mediante su obra Liber Abaci (Libro de los Ábacos o Libro de los Cálculos)

Dentro del conjunto de números existen diversos subconjuntos. Los números naturales son los primeros que utiliza el ser humano, se definen como los enteros no negativos y se representan por la letra $\mathrm{N}$. Los números enteros no poseen principio ni fin, contienen al conjunto de números naturales, a los números enteros negativos y al cero. Se representan por la letra Z (zahl en alemán significa número o cantidad), son realmente un subconjunto de los números racionales o fracciones ya que todo número entero tiene como denominador a la unidad. El cero es neutro, ni positivo ni negativo.

Los números racionales son las fracciones, los decimales finitos y los decimales infinitos periódicos. Se representan por la letra $\mathrm{Q}$ (de quotient en varios idiomas europeos).Cumplen la propiedad arquimediana o de densidad, es decir que para cualquier pareja de números racionales existe otro número racional situado entre ellos. Los números irracionales se denominan así porque no se pueden escribir en forma de fracción. Son los números que no tienen una raíz exacta. Se representan por Q'. Son un subconjunto de los números reales. Parece que fue Hipaso, alumno de la Escuela Pitagórica quien los describió en primer lugar al escribir la raíz de 2 en forma de fracción. 
Los números reales se sistematizan en el siglo XIX con las aportaciones de Georg Cantor y Richard Dedekind. Se representan por la letra R. Son el subconjunto de los números complejos, y contienen a los subconjuntos de $\mathrm{N}$, $Z$, Q y Q'. Poseen una expresión decimal, e incluyen tanto a los números racionales $(\mathrm{Q})$, los irracionales $\left(\mathrm{Q}^{\prime}\right)$, algebraicos y trascendentales.

Aunque descubiertos en la Antigua Grecia, los números complejos renacen en el desarrollo de las ecuaciones cuadráticas que generan raíces cuadradas de números negativos. Carl F. Gauss los describió en 1831 bajo la forma $a+b i$. Se representan por la letra $C$ y es el conjunto de números que contiene a todos los subconjuntos del resto de números.

$$
\mathrm{C}=\left\{\mathrm{N}, \mathrm{Z}, \mathrm{Q}, \mathrm{Q}^{\prime}, \mathrm{R}\right\}
$$

\section{Georg Cantor y el infinito.}

La naturaleza del infinito ha sido siempre objeto de controversia. Las paradojas de Zenón de Elea(4) suscitaron ya el problema en la antigüedad. Zenón explicaba con inquietante lucidez que el movimiento es imposible, porque exige que el móvil pase por una infinidad de puntos en un tiempo finito. El éxito de la física newtoniana es en gran parte consecuencia del cálculo de tasas de variación de lo infinitamente pequeño, y ello a pesar de que durante más de 200 años no pudo ofrecerse una formulación matemáticamente rigurosa de esta idea. En tiempos modernos han aparecido nuevos problemas asociados con el infinito en la teoría de conjuntos abstractos, teoría que proporciona fundamento y cimentación a prácticamente la totalidad de las matemáticas contemporáneas.

Además, la idea de infinito ha estado siempre, a través de la historia, cargada de tintes y matices teológicos, que han pesado en la aceptación o en el rechazo de este concepto y de las doctrinas matemáticas o filosóficas con él asociadas. Todas estas corrientes de pensamiento convergen en la obra del matemático Georg Cantor (1845-1918)

Al desarrollar la que él mismo bautizó "aritmética de los números 
transfinitos", dotó de contenido matemático al concepto de infinito actual. Y al hacerlo así puso los cimientos de la teoría de conjuntos abstractos, contribuyendo además a fundamentar el cálculo diferencial y el continuo de los números reales. El más notable logro de Cantor consistió en demostrar, con rigor matemático, que la de infinito no era una noción indiferenciada. No todos los conjuntos infinitos son de igual tamaño; por consiguiente, es posible establecer comparaciones entre ellos. El conjunto de todos los puntos de una recta, por ejemplo, y el conjunto de todos los números fraccionarios son, ambos, conjuntos infinitos.

Demostró que, en un sentido bien definido, el primero de tales conjuntos es de tamaño mayor que el del segundo (Figura no 3 )

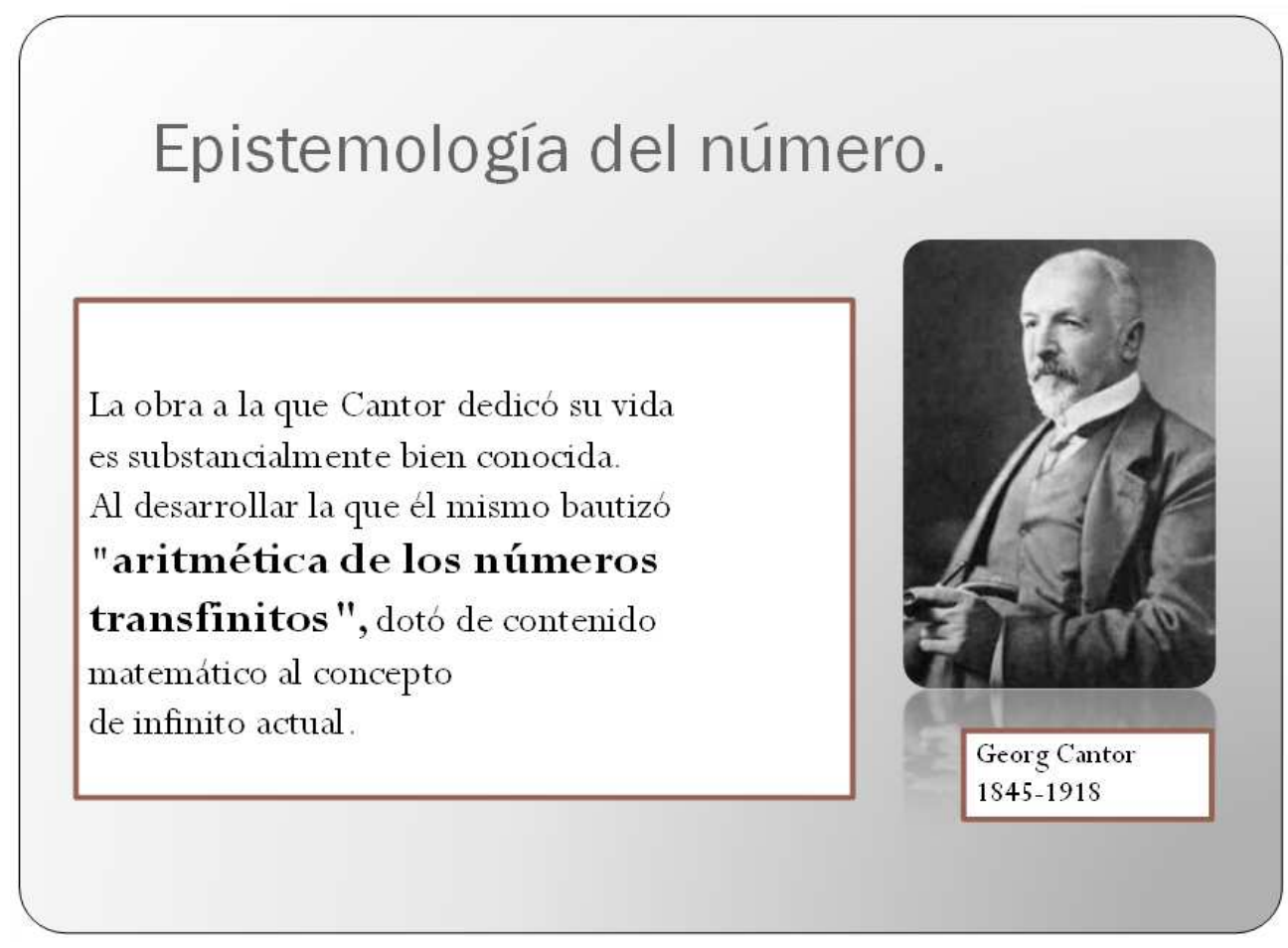

Figura $n^{\circ} 3$.

Cantor y la aritmética de los números transfinitos.

Las ideas de Cantor resultaron tan chocantes a la intuición de sus contemporáneos, que el eminente matemático francés Henri Poincaré condenó la teoría de números transfinitos como una "enfermedad", de la que algún día llegarían las matemáticas a curarse. Leopold Kronecker, que fue uno de los maestros de Cantor, y miembro preeminente de la matemática institucional alemana, llegó incluso a atacarle directa y personalmente, calificándolo de "charlatán científico", " renegado" y "corruptor de la juventud". No es motivo 
de este artículo analizar el Teorema de Cantor sobre la cardinalidad del conjunto potencia, ni la Hipótesis del Contínuo (HC) que versa sobre la cardinalidad de los números reales. Tan sólo queremos subrayar la importancia de este investigador en la historia de la ciencia posterior y su incomprensión social ${ }^{(5)}$

\section{Kurt Gödel y la aritmética.}

Gödel (1906-1978) intentó razonar el fundamento de las matemáticas desde la lógica y desde la teoría de conjuntos. Sus Teoremas de la Incompletitud fueron publicados en el año 1931 mucho antes de enseñar en Princeton (USA)

El más célebre de ellos, afirma que para todo sistema axiomático recursivo auto-consistente lo suficientemente poderoso como para describir la aritmética de los números naturales, existen proposiciones verdaderas sobre los números naturales que no pueden demostrarse a partir de los axiomas. Para desarrollar este teorema ideó una técnica denominada numeración de Gödel, codificando expresiones formales como números naturales. Lo que nos quiso señalar Gödel es que incluso la aritmética más elemental tiene vericuetos lógicos inconsistentes (Figura no 4)

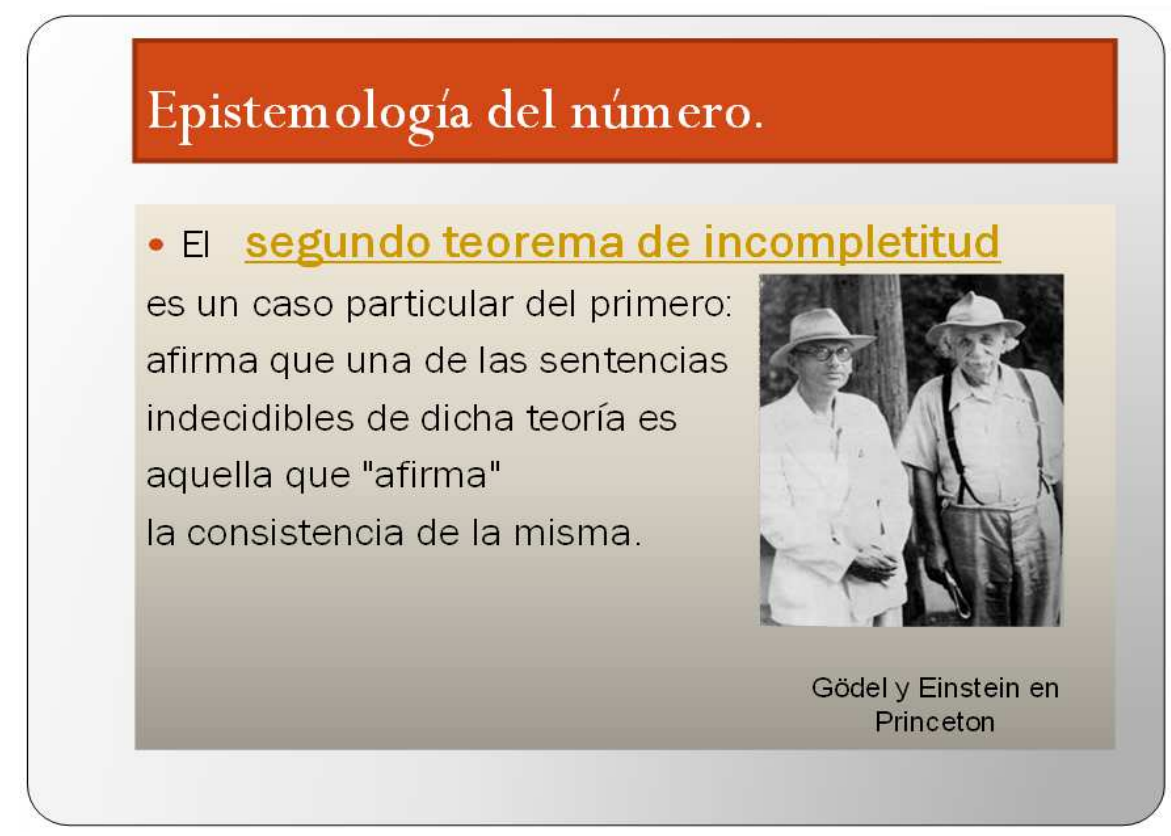

Figura no 4.

Gödel y Einstein coincidieron trabajando en la ciudad norteamericana de Princeton. 


\section{Incertidumbre alrededor del concepto de número.}

Hemos visto de forma bastante sintética, como la obra de Cantor y de Gödel ha introducido una fuerte dosis de incertidumbre en el sacrosanto concepto de número que la sociedad occidental había hecho suyo desde la Escuela Pitagórica. Cuantificar y analizar una realidad con conceptos puramente matemáticos no lo es todo. Hay aspectos de nuestro entorno real que no se pueden aquilatar a través del número. No queremos entrar en este momento en las discusiones existentes entre el paradigma frecuentista y el bayesiano en la bioestadística contemporánea(6)(7) ni tampoco en las dificultades de aplicación metodológica de la salud basada en la evidencia (8)(9) (10) para el análisis de la práctica clínica, Para nosotros, todas estas incertidumbres dentro del ámbito cuantitativo, las intenta cubrir la investigación cualitativa haciéndola, de esta forma, necesaria en la investigación sanitaria (Figura no 5)

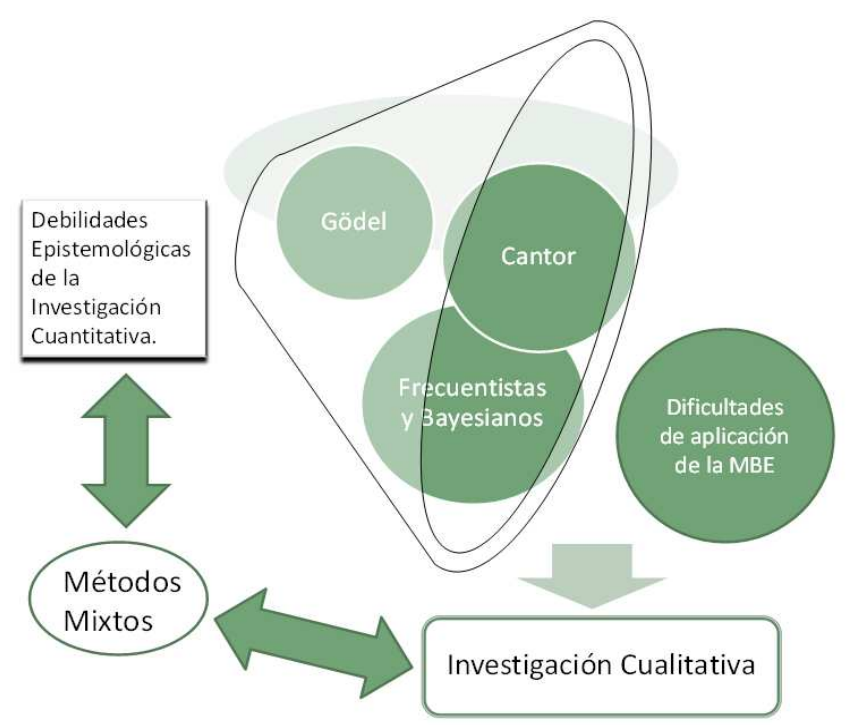

Figura $n^{0} 5$.

Removiendo la vasija de las dudas epistemológicas de la investigación cuantitativa se obtiene la necesidad de la investigación cualitativa y la metodología mixta.

\section{Un método alternativo.}

La investigación cualitativa puede entenderse como una categoría de 
diseños que extraen descripciones a partir de observaciones y que adoptan la forma de entrevistas, narraciones, notas de campo, grabaciones, transcripciones de audio, video casetes, registros escritos de todo tipo, fotografías, películas o artefactos. Según Lincoln y Denzin ${ }^{(11)}$, la investigación cualitativa es un campo interdisciplinar, transdisciplinar y en ocasiones contradisciplinar.

Desde una perspectiva sociológica se puede afirmar que la investigación cualitativa comenzó en los Estados Unidos a principios del siglo XX con la preocupación por la urbanización y la inmigración de grandes masas de personas y su impacto en el sector salud. Las raíces antropológicas habría que buscarlas en el trabajo de los primeros antropólogos evolucionistas de la segunda mitad del siglo XIX. Los estudios etnográficos anteriores a esa época no tenían análisis de campo. Los dos abordajes epistemológicos más clásicos son la teoría fundamentada y la inducción analítica ${ }^{(12)}$.

\section{Evidencia y Calidad de la Investigación Cualitativa.}

El concepto de evidencia se asocia al manejo de información compleja apoyada en procedimientos metodológicos de análisis sistemáticos, estructurados y con una validez comprobada, comúnmente asociada a la investigación biosanitaria basada en la evidencia. La salud basada en la evidencia, a pesar de la dificultad inherente, es capaz de ofrecer resultados con impacto científico.

En la actualidad se discute la posibilidad de extrapolar esta concepción a las investigaciones de síntesis cualitativa, siempre considerando criterios fundamentales de este tipo de investigación y que pueden definir la evidencia cualitativa de la siguiente forma: a)La evidencia cualitativa está sujeta a las características propias de la investigación primaria, por lo cual los conceptos definidos como subjetivos en las investigaciones primarias se traspasan al proceso de síntesis de la información, b) La evidencia cualitativa en salud va a depender del mecanismo con que se evalúe la investigación primaria, siendo 
ésta clasificada mediante guías de lectura crítica o por medio de técnicas de síntesis de la información ${ }^{(13)}$.

Realmente es complicado entender un nexo de unión entre el concepto de evidencia que es neopositivista ${ }^{(8)}{ }^{(9)}$ y la investigación cualitativa que goza de unas características epistemológicas menos rígidas. Nosotros afirmamos que tal vínculo existe aunque su demostración no sea el objetivo de este artículo. Reynolds y colaboradores ${ }^{(14)}$ realizaron un análisis muy interesante sobre la calidad de un conjunto de publicaciones en investigación cualitativa. Tras aplicar criterios de inclusión y exclusión seleccionaron un total de 37 artículos. En sólo 10 de ellos, los autores especificaban la posición epistemológica desde la que habían realizado el trabajo.

Cinco artículos se definían como postpositivistas-realistas y otros cinco como interpretativo-constructivistas. La perspectiva postpositivista-realista afirma que la realidad social existe, pero que nunca puede ser totalmente aprehendida y que los métodos deben de ser sistemáticos y rigurosos. La óptica interpretativo-constructivista niega la existencia de la realidad y afirma que el conocimiento se construye a través de la investigación y de la interpretación, siendo esta última dependiente de los valores y de las asunciones del investigador.

\section{Metodología Mixta.}

En todo caso abogamos por la posibilidad de utilizar ambos acercamientos de forma conjunta, por ejemplo el cualitativo como complemento del cuantitativo ${ }^{(15)}$ o viceversa(16).

Las fortalezas de la investigación cuantitativa (la exactitud y medida de un constructo específico, la posibilidad de realizar comparaciones grupales, el cuantificar la fuerza de asociación entre las variables de interés y la capacidad de crear un modelo para probar una hipótesis) se ven contrapesadas por la falta de análisis del ser humano real ("decontextualización") y por las que hemos expuesto anteriormente en este ensayo. Contra esas características, la 
investigación cualitativa presenta un mayor aspecto de "contextualización" con capacidad de analizar extensas narraciones personales de experiencias descritas en su escenario original ${ }^{(17)}$. Nosotros pensamos, por ello, que el conjunto de ambas es un instrumento muy útil para la comprensión de la realidad.

\section{Conclusión.}

A partir de las aportaciones de Cantor y de Gödel a la filosofía de la ciencia, los cimientos epistemológicos del concepto matemático de número se han cimbreado. La incertidumbre que crean esos avances junto con la discusión frecuentista-bayesiana en el campo de la estadística y los inconvenientes metodológicos de la salud basada en la evidencia, hacen totalmente necesaria la investigación cualitativa en salud (Figura no 5). Es más, creemos necesaria la definición epistemológica en cada trabajo de investigación cualitativa para afianzar su calidad(18). Los métodos mixtos contrapesan las desventajas de las dos aproximaciones a la realidad. En todo caso este trabajo es fruto de la actitud continua de reflexividad que debe presidir cualquier tipo de investigación ${ }^{(19)}$.

\section{Bibliografía.}

1. Borwein, J M, Borwein, $P$ B: Ramanujan y el número pi. Revista Investigación y Ciencia (edición española). 1988; 139: 72-80.

2. Berlinski , D : Ascenso infinito. Breve historia de las matemáticas. Random House Mondadori. 2006. Barcelona. Páginas: 15-16.

3. Leonardo de Pisa: http://es.wikipedia.org/wiki/Leonardo de Pisa Visitado el 8 de Mayo de 2014.

4. Ortega Calvo, M: Apuntes sobre Filosofía de la Ciencia e Investigación Clínica. Wanceulen Médica. Sevilla. 2010. Páginas: 67-68. 
5. Mosterín , J : Los Lógicos. Espasa-Calpe. Madrid. 2000. Páginas: 89-135.

6. Silva Ayçaguer, LC, Muñoz Villegas, A: Debate sobre métodos frecuentistas vs bayesianos. Gac Sanit $2000 ; 14: 482-494$. Disponible en:

http://lcsilva.sbhac.net/Articulos/7.Debate sobre metodos frecuentistas vs bayesianos.pdf

7. Sarria Castro, M, Silva Ayçaguer, LC: Las pruebas de significación estadística en tres revistas biomédicas: una revisión crítica. Rev. Panam. Salud Publica. 2004;15:300-6. Disponible en: http://www.scielosp.org/pdf/rpsp/v15n5/22001.pdf

8. Ortega Calvo, M, Cayuela Domínguez, A: Medicina basada en la evidencia: Una crítica filosófica sobre su aplicación en atención primaria. Rev Esp Salud Pública 2002; 76: 115-120. Disponible:

http://www.msssi.gob.es/biblioPublic/publicaciones/recursos propios/resp/revista cdrom/vol7 6/vol76 2/RS762C 115.pdf

9. Morales Asencio, JM, Gonzalo Jiménez , E , Martín Santos ,FJ , Morilla Herrera , JC: Salud Pública basada en la evidencia. Recursos sobre la efectividad de intervenciones en la comunidad. Rev Esp Salud Pública 2008; 82: 5-20. Disponible en: http://scielo.isciii.es/pdf/resp/v82n1/colaboracion1.pdf

10. González-Torrente S, Pericas-Beltrán J, Bennasar-Veny M, Adrover-Barceló R, Morales-Asencio JM, De Pedro-Gómez J: Perception of evidence-based practice and the professional environment of Primary Health Care nurses in the Spanish context: a cross-sectional study. BMC Health Serv Res. 2012; 12:227. Disponible: http://www.biomedcentral.com/content/pdf/1472-6963-12-227.pdf

11. Denzin, N K , Lincoln , Y S : Handbook of Qualitative Research . $2^{\text {nd }}$ ed. Thousand Oaks. Sage. CA. USA.

12. Amezcua, M, Gálvez Toro , A : Los modos de análisis en investigación cualitativa en salud : perspectiva crítica y reflexiones en voz alta. Rev. Esp. Salud Pública 2002; 76: 423-436. Disponible en: http://www.msssi.gob.es/biblioPublic/publicaciones/recursos propios/resp/monografico2/RS765 423.pdf

13. Oliva, $\mathrm{P}$, Buhring , $\mathrm{K}$ : Investigación cualitativa y evidencia en salud: Respuestas fundamentales para su comprensión. Rev. Chil. Salud Pública. 2011; 15: 173-179. Disponible en:

http://www.revistasaludpublica.uchile.cl/index.php/RCSP/article/viewFile/17714/18474 
14. Reynolds J, Kizito J, Ezumah N, Mangesho P, Allen E, Chandler C : Quality assurance of qualitative research: a review of the discourse. Health Res Policy Syst. 2011; 9: 43. Disponible en:

\section{http://www.health-policy-systems.com/content/pdf/1478-4505-9-43.pdf}

15. Alvarez del Arco, D, Rodríguez Riero, C, Sanchidrián de Blas ,C et al : Aportaciones e inconvenientes de la incorporación de metodología cualitativa en la evaluación de servicios sanitarios. Un caso práctico: la evaluación de una consulta de alta resolución. Rev Calid Asist. 2012 ; 27 : 275-282.

16. González-García L, Chemello C, García-Sánchez F, et al :Aphorisms and short phrases as pieces of knowledge in the pedagogical framework of the Andalusian School of Public Health. Int J Prev Med. 2012 ; 3 : 197-210. Disponible en: http://ijpm.mui.ac.ir/index.php/ijpm/article/view/288/405

17. González - Castro F, Kellison J G, Boyd SJ, Kopak A: A Methodology for Conducting Integrative Mixed Methods Research and Data Analyses. J Mix Methods Res. 2010 ; 4: 342 - 360. Disponible en: http://www.ncbi.nlm.nih.gov/pmc/articles/PMC3235529/pdf/nihms-248033.pdf

18. Ortega Calvo, M, Román Torres, P, Lapetra Peralta J : La epistemología como propedéutica de la investigación sanitaria. Gac Sanit. 2011; 25 : 79-83. Disponible en : http://scielo.isciii.es/pdf/gs/v25n1/especial.pdf

19. De la Cuesta Benjumea, C: La reflexividad: un asunto crítico en la investigación cualitativa. Enferm Clin. 2011; 21:163-167. 